\title{
Brain-Computer Interface Technique for Electro-Acupuncture Stimulation Control
}

\author{
Dong Ming*, Yanru Bai, Xiuyun Liu, Xingwei An, \\ Hongzhi Qi, Baikun Wan \\ Department of Biomedical Engineering \\ Tianjin University \\ Tianjin, China \\ *: richardming@tju.edu.cn
}

\begin{abstract}
Electro-acupuncture stimulation (EAS) technique applies the electrical nerve stimulation therapy on traditional acupuncture points to restore the muscle tension. The rapid rise and development of brain-computer interface (BCI) technology makes the thought-control of EAS possible. This paper designed a new BCI-controls-EAS (BCICEAS) system by using event related desynchronization (ERD) of EEG signal evoked by imaginary movement. The Fisher parameters were extracted from feature frequency bands of EEG and classified into EAS control commands by Mahalanobis Classifier. A feedback training technique was introduced to enhance the signal feature through a visual feedback interface with a virtual liquid column, which height varied along with EEG power spectral feature. Experimental results demonstrated the validity of the proposed method, including the effective improvement of feedback training on signal feature and reliable control of EAS. It is hoped the BCICEAS can explore a new way for EAS system design and help people who sufferers with severe movement dysfunction.
\end{abstract}

Keywords-electro-acupuncture stimulation; brain-computer interface; event related desynchronization; imaginary movement; Mahalanobis classifier

\section{INTRODUCTION}

Acupuncture has been used for restoring the muscle tension in the Orient for thousands of years, with recognition as a complementary medicine by NIH Consensus in 1997 [1,2]. Electro-acupuncture stimulation (EAS) is widely used as a substitute for classical acupuncture. It has recently been demonstrated in humans that EAS produces reflex changes in muscle motor nerve activity and clinical reports of successful treatment of patients with a variety of symptoms give this method high credibility [3-4].

Traditional EAS systems would be an unfeasible solution for those severe paraplegic or hemiplegic patients without voluntary control of upper limbs. Nowadays, the rapid rise and development of brain-computer interface (BCI) technology makes the free-hand thought-control of EAS possible. BCI provides alternative communication and control channels to convey messages and commands from the brain to the external world, with the aim of assisting, augmenting, or repairing human cognitive or sensory-motor functions, especially for those patients with severe neurological or muscular diseases [5-11].

\author{
Yong $\mathrm{Hu}$, KDK Luk \\ Department of Orthopaedics and Traumatology \\ University of Hong Kong \\ Hong Kong, China
}

This study proposed a BCI-controls-EAS (BCICEAS) system to stimulate upper limbs action based on event related desynchronization (ERD) of EEG signal evoked by imaginary movement. A feedback training technique was designed to enhance relevant EEG feature through a visual interface with a virtual liquid column, which height varied along with EEG power spectral feature.

\section{CONTROL PLATFORM OVERVIEW}

The control platform of BCICEAS to stimulate upper limbs action in this study includes three modules: signal acquisition module, signal processing module and EAS stimulator module, as shown in Fig. 1.

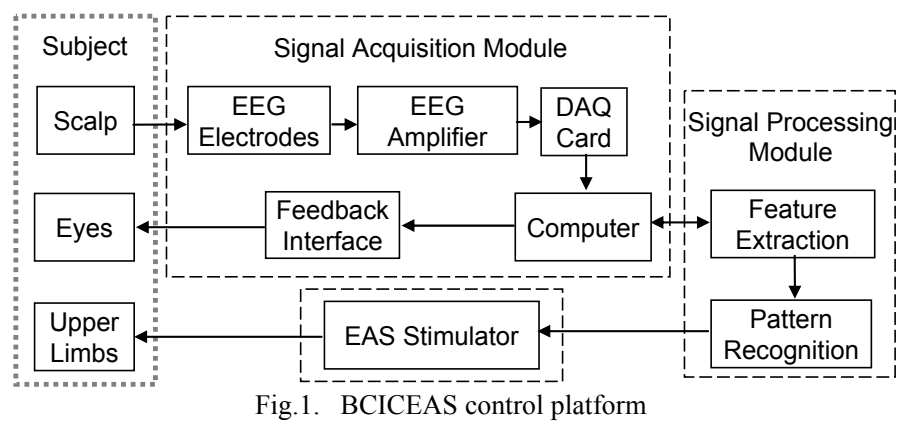

\section{A. Signal Acquisition Module}

EEG signal was collected from C3 and C4 of 10-20 international electrode placement, while $\mathrm{A} 1$ and $\mathrm{A} 2$ on lift and right earlobes as reference electrodes[12]. The 9216SM (Symtop Co, Ltd., China) is used as EEG amplifier and USB6125 DAQ card (National Instrument Co, Ltd., USA) realized data input to computer throughs analog-to-digital conversion. Fig. 2 shows the experimental feedback interface, which aim is to induce human brain to generate corresponding EEG signal when the user performs imagining or no imagining action in accordance with the system's cues and adjust the imaginary level through the virtual liquid column representing EEG power spectral feature.

\section{B. Signal Processing Module}




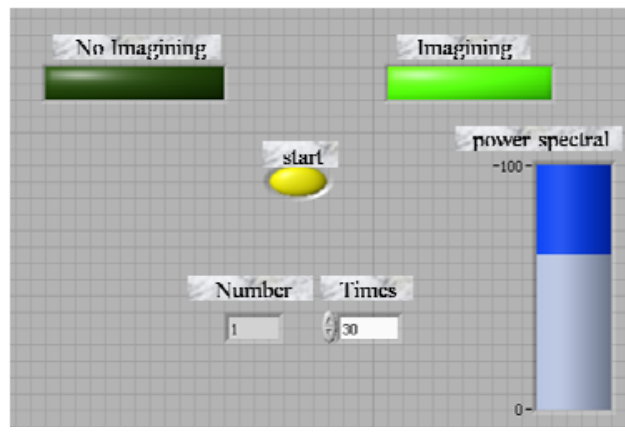

Fig.2. Feedback interface

The signal processing module includes the algorithms of feature extraction and pattern recognition, which are key factors to enhance the reliability and performance of BCICEAS system and will be introduced in Part 3 in details.

\section{EAS Stimulator Module}

EAS stimulator module is a rectangular pulse generator as shown in Fig. 3.

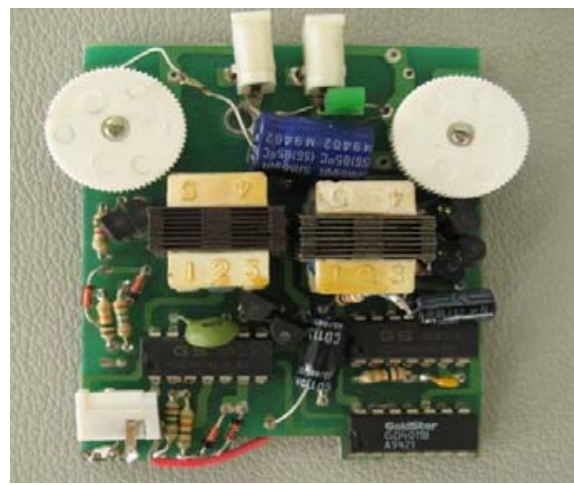

Fig.3. Integrated circuit of EAS stimulator

\section{SIGNAL PROCESSING METHOD}

The study of nerve electrophysiology showed that either an actual body movement or just an imagination of the movement can raise obvious intensity changes in the power spectrum density (PSD) of some characteristic band components in evoked potentials [13]. The decrease of power spectrum ratio is called as event related desynchronization (ERD), while the increase is called as event related synchronization (ERS). In addition, both the characteristic bands and spatial cortex regions of ERD/ERS is related to the kind of actual or imaginary movement. This means that the ERD/ERS caused by the brain imaginary movements can be used as an effective means for EEG feature detection and pattern recognition.

ERD/ERS has many definition and calculation methods, such as Hilbert transformation [14], band power spectrum [15], and power spectral decomposition [16]. Herein, we adopt the definition of band power spectrum proposed by Pfurtschellera and Aranibar [17].

$$
C_{E R D / E R S}=\frac{A-R}{R} \times 100 \%
$$

where $A$ and $R$ stand for the PSD during the response period after and before evoked stimulation, respectively.

\section{A. Feature Extraction}

1) Time-Frequency Analysis: Considering that ERD/ERS signals have specific frequency bands and a fixed time delay compared to the evoked stimulation, we introduce the method of time-frequency analysis based on short time Fourier transform (STFT) to analyse the change in density distribution of PSD before and after the stimulation [18].

STFT is the major method used for time-frequency analysis at present, which is defined as follows:

$$
\operatorname{STFT}_{x}(\omega, t)=\int_{-\infty}^{\infty} x\left(t^{\prime}\right) w^{*}\left(t^{\prime}-t\right) e^{-j \omega t^{\prime}} d t^{\prime}
$$

where $\omega$ means the angular frequency, and $w(t)$ stands for the window function, $w^{*}\left(t^{\prime}-t\right)$ is the complex conjugate function of $w\left(t^{\prime}-t\right)$.

\section{2) Fisher Parameters:}

As one of the most effective measures of class separability, Fisher analysis is widely used in pattern recognition [19]. In our research, matching pursuit (MP) algorithm is firstly used to acquire the time-frequency distribution of EEG feature set. Then we get Fisher parameters according to the Fisher criterion. The MP-Fisher parameter can be calculated with the following formula:

$$
S(t, f)=\frac{\left[M_{t f(1)}(t, f)-M_{t f(2)}(t, f)\right]^{2}}{S_{t f(1)}(t, f)+S_{t f(2)}(t, f)}
$$

where $M_{t f(1)}(t, f)$ and $M_{t f(2)}(t, f)$ stand for the average of time-frequency distribution feature of two classes, while $S_{t f(1)}(t, f)$ and $S_{t f(2)}(t, f)$ are the variances of them. The bigger $S(t, f)$ is, the higher the separability is. Zero value of $S(t, f)$ means that the features of them are impartibility.

Fisher analysis can reveal the differences in PSD between one class and the others. That is to say, with enough large Fisher coefficients, the frequency bands and time periods can be considered as the features of this class, which should be extracted for classification.

\section{B. Pattern Recognition}

A discriminant analysis method of Mahalanobis distance was introduced to identify and classify the feature parameters extracted from the EEG signals evoked by imaginary movement. Assume that $G_{1}$ and $G_{2}$ are two classes, and the Mahalanobis distance between $x$ and these two classes are defined as follow:

$$
\begin{aligned}
& d^{2}\left(x, G_{1}\right)=\left(x-u^{(1)}\right)^{T} \sum_{1}^{-1}\left(x-u^{(1)}\right) \\
& d^{2}\left(x, G_{2}\right)=\left(x-u^{(2)}\right)^{T} \sum_{2}^{-1}\left(x-u^{(2)}\right)
\end{aligned}
$$


where $u^{(1)}$ and $u^{(2)}$ stand for the average of $G_{1}$ and $G_{2}$, while $\Sigma_{1}$ and $\Sigma_{2}$ stand for the covariance of $G_{1}$ and $G_{2}$, respectively.

We construct the discriminant formula as follow:

$$
w(x)=\frac{d^{2}\left(x, G_{1}\right)-d^{2}\left(x, G_{2}\right)}{2}
$$

where $x \in G_{1}$, if $w(x)<0 ; x \in G_{2}$, if $w(x)>0$; $x$ is pending, if $w(x)=0$.

\section{TRAINING AND SYSTEM EXPERIMENTAL RESULTS}

\section{A. Training Experimental Results}

EEG training experiment was induced by imaginary movements. There are two groups of the recruited healthy adult subjects in the experiments, one group with feedback training $(\mu=22.4 \pm 3.5$ years $)$ and another group without feedback training $(\mu=21.7 \pm 4.1$ years). Each group has 6 subjects with 3 males and 3 females. There are three stages for each subject's experiment. Each stage lasts about 40 minutes and comprises of four sets (30 trials of motor imagination for one set). The rest time between two trial sets is about three minutes and each trial lasts about nine seconds.

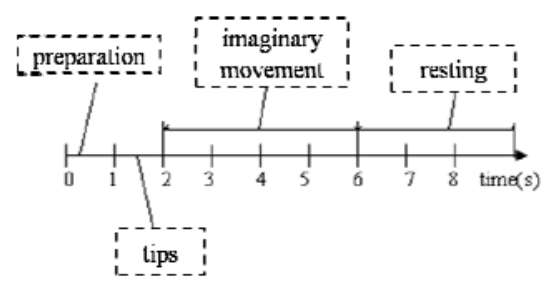

Fig.4. Trial timing paradigm

The trial timing paradigm is shown in Fig. 4. The first second during trial beginning is the period of preparation and the subject keeps quite. At $t=1$ second, a signal light will be lit to indicate imagining or no imagining task. At $t=2$ second, a signal light will be lit to indicate task start and, in the next 4 seconds, the subject is asked to perform the imaginary movement (imaginary lift of the upper limb) or just keep quite according to the tip of signal light at $t=1$ second. From $t=6$ to 9 seconds is a resting period, when all signal lights will black out, and the subject keeps quite and prepares for next trial.

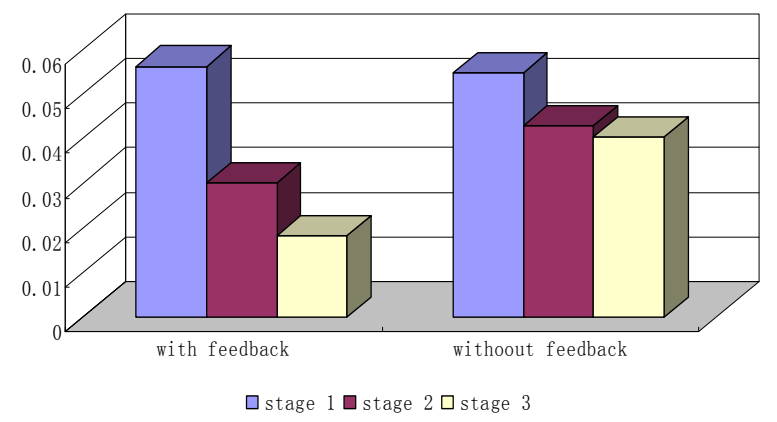

Fig.5. Comparison of the mean of ERD value
For the group of subjects with feedback, the imaginary state can be adjusted through a visual feedback of a virtual liquid column representing EEG power spectral value on feedback interface as shown in Fig.2. Firstly, the characteristic frequency bands of each subject are extracted according to Fisher parameter criterion. Then the PSD is averaged in the corresponding characteristic bands and time periods. Finally, after the data is normalized, we can calculate the ERD value of each imaginary movement according to formula (1).

By calculating the mean of ERD value in each stage, we can see that the mean of ERD value is from 0.0561 in stage 1 down to 0.0181 in stage 3 for the group with feedback, while from 0.0548 down to 0.0403 for the group without feedback, as shown in Fig. 5. As mentioned above, the ERD value is defined as the ratio of the difference between imagination and resting period. The smaller the ERD value is, the higher the EEG feature of imaginary movement is. So EEG feature has been greatly enhanced after training both with and without feedback. It is also concluded that feedback training has a more enhancement of EEG feature in comparison to no feedback training.

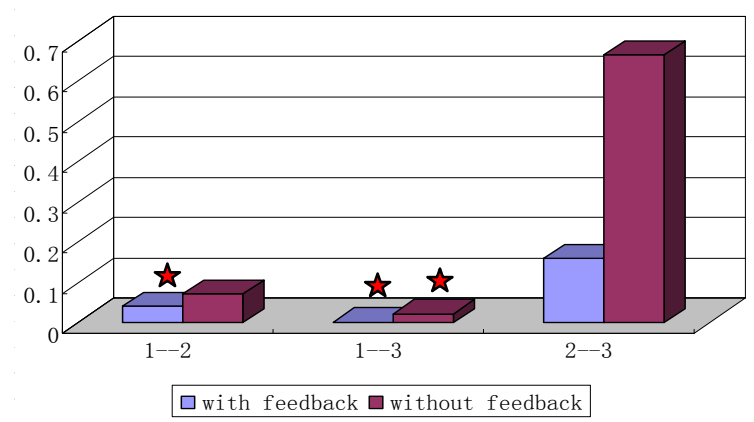

Fig.6. $\mathrm{P}$ value in t-test

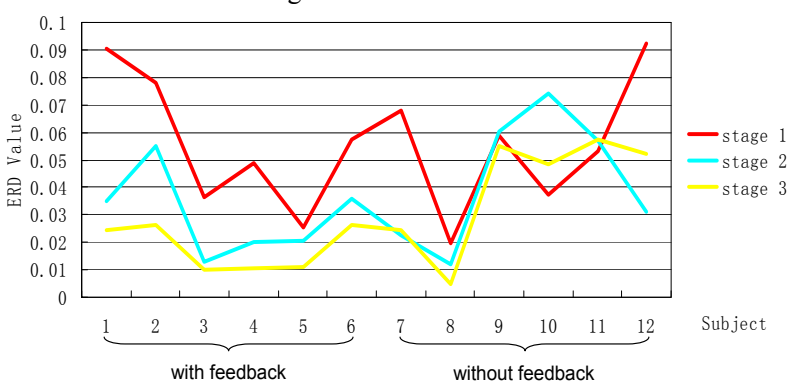

Fig.7. The curve of the mean of ERD value by feedback and without feedback

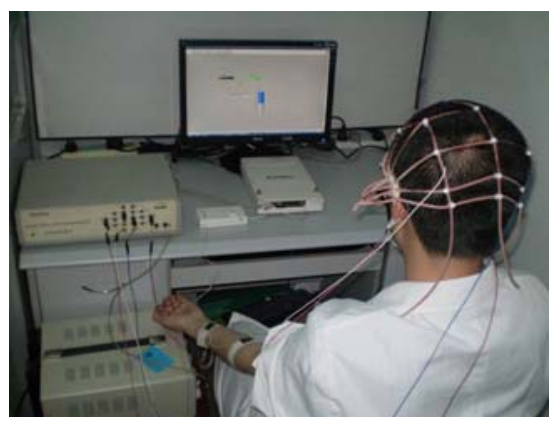

Fig.8. BCICEAS system experimental environment 
By comparing the ERD value of two stages through $t$-test, we get the $P$ value which reflects the degree of difference, as shown in Fig. 6. Significant difference exists when $P$ is less than 0.05 , as marked with red star. For the group with feedback, the differences between stage 1 and next two stages are significant. For the groups without feedback, only the differences between stage 1 and 3 are significant. For stage 1, there is a problem of strangeness to influence imaginary efficiency because of the first use of system. Therefore, feedback training process is necessary for BCICEAS system to overcome this problem. It seems having a saturation phenomenon when training from stage 2 to 3 . This reflects the advantages of no long training process with the BCICEAS system, which is very suitable for medical rehabilitation.

Fig. 7 shows the curves of the mean of ERD value for subjects with and without feedback. In comparison to the group without feedback, there is a more obvious trend of ERD value along with increasing stage in the group with feedback. These results reveal that the feedback training can effectively enhance the intensity of EEG feature.

\section{B. BCICEAS System Test Results}

The effectiveness of the training technique has been verified through the previous experiment. However, our ultimate goal is to achieve successful control of external equipment (EAS). So an on-line system test is carried out on three subjects with feedback training by connecting the control command to the EAS driving port. Fig. 8 shows the real experimental environment. The electrodes are placed in the acupuncture Qu-Ze and Nei-Guan, which are the sensitive points of stimulation for lifting the fore-arm.

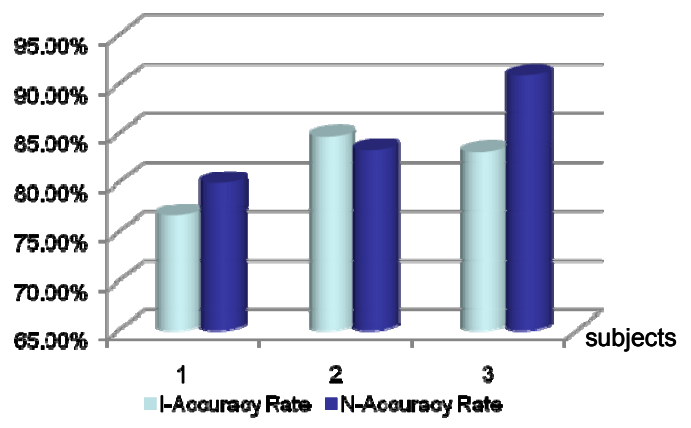

Fig.9. The highest accuracy rate of the testing experiment

We define the accuracy rate as follows: I-Accuracy Rate means the upper limb is successfully lifted by stimulating of EAS if the system instructs subject to imagine; N-Accuracy Rate means the subject keeps quite as expected if the system instruct not to imagine. Fig. 9 shows the I-Accuracy Rates and $\mathrm{N}$-Accuracy Rates of the experiments on three subjects. We can see that the accuracy rates of these three subjects all reach more than $76.7 \%$ and the third one reach even more than $90 \%$, which proves that our BCICEAS system has a reliable operability under the effect of feedback training.

\section{CONCLUSION}

In conclusion, the BCICEAS system designed in this paper is demonstrated feasible to use subject's thought to control electro-acupuncture stimulation to resume action ability of upper limbs. The feedback training technique is also verified to effectively improve the system performance. The method may be further used for serving more disabled people to recover their lost functions.

\section{ACKNOWLEDGMENT}

This research was supported by National Natural Science Foundation of China (No.30970875, No.90920015 and No. 60501005), Joint Project of National Natural Science Foundation of China-Royal Society of Edinburgh of UK (No. 30910494/C1009), The National High Technology Research and Development Program of China (No.2007AA04Z236), Intentional Cooperation Key Program (No.08ZCGHHZ00300) and Biomedical Engineering Key Program (No. 07ZCKFSF01300) of Tianjin Science Technology Support Plan.

\section{REFERENCES}

[1] J. Am. Med. Assoc. NIH Consensus Conference, Acupuncture, p.1518$1524,1998$.

[2] V. Clement-Jones, L. McLoughlin, S. Tomlin, G.M. Besser, L.H. Rees, H.L. Wen, "Increased beta-endorphin but not met-enkephalin levels in human cerebrospinal fluid after acupuncture for recurrent pain", Lancet 2 , pp.946-949,1980.

[3] J.H. Lee, A.J. Beitz, "The distribution of brain-stem and spinal cord nuclei associated with different frequencies of electroacupuncture analgesia”, Pain 52, pp.11-28, 1993.

[4] J.M. Peets, B. Pomeranz, "CXBK mice deficient in opiate receptors show poor electroacupuncture analgesia", Nature 273, pp.675-676, Nov. 1978.

[5] W.T. Zhang, Z. Jin, G.H. Cui, K.L. Zhang, L. Z hang, Y.W. Zeng, F. Luo, A.C. Chen, J.S. Han, "Relations between brain network activation and analgesic effect induced by low vs. high frequency electrical acupoint stimulation in different subjects: a functional magnetic resonance imaging study", Brain Research, 982 (2), pp.168 178, 2003.

[6] J.R. Wolpaw, N. Birbaumer, W.J. Heetderks, D.J. McFarland, P.H. Peckham, G. Schalk, E. Donchin, L.A. Quatrano, C.J. Robinson, T.M. Vaughan, "Brain-computer interface technology: A review of the first international meeting”, IEEE Trans.Rehabil.Eng., vol.8(2), pp.222-225, 2000 .

[7] T.M. Vaughan, J.R. Wolpwa, E. Donchin, "EEG-based communication: prospects and problems", IEEE Trans.Rehabil.Eng., vol.4(4), pp.425$430,1996$.

[8] J.R. Wolpaw, N. Birbaumer, D.J. McFarland, G.P furtscheller, and T.M. Vaughan, "Brain-computer interfaces for communication and control", Clin. Neurophysiol, vol.113(6), pp. 767-791, 2002.

[9] J.R. Wolpaw, N. Birbaumer, D.J. McFarland, G.P furtscheller, and T.M. Vaughan, "Brain-computer interfaces for communication and control", Clin Neurophysology, vol.113(6), pp.94-109, 2002.

[10] B.K. Wan, Y. Gao, L. Zhao, H.Z. Qi, "Brain-computer interface: A new channel for information communicate between human brain and environments", Bio-medical engineering foreign medical sciences, vol.28, pp.4-9, 2005.

[11] T.M. Vaughan, "Brain-Computer Interface technology: a review of the second international meeting", IEEE Trans. Neural. Syst. Rehabil .Eng, vol.11, pp.94-109, 2003.

[12] The International 10-20 System Electrode Placement. [Online]. http://www.telemedx.com/ protocol/section7.2.html. 
[13] P. Zhuang, "EEG event-related resynchronization and synchronization of activities and sports related operation", Chinese Journal of Clinical Rehabilitation, vol.8, pp.152-154, 2004.

[14] M.B. Sterman, D.A. Kaiser, B. Veigel, "Spectral analysis of eventrelated EEG responses during short-term memory performance", Brain topography, vol.9, pp.21-30, 1996.

[15] A.P. Burgress, J.H. Gruzelier, "The reliability of event-related desynchronization: a generalisability study analysis", International Journal of Psychophysiology, vol.23, pp.163-169, 1996.
[16] G. Florian, G. Pfurtscheller, "Dynamic spectral analysisi of event-related EEG data", Electroencephalography and Clinical Neurophysiology, vol.95, pp.110-117, 1995.

[17] G. Pfurtscheller, F.H. Lopes da Silva, "Event-related EEG/MEG synchronization and desychronization: basic principles", Clin Neurophysiology, vol.110, pp.1842-1857, 1999.

[18] L. Liu, B.G. Hao, "Time-frequency analysis theory and application", Computer automatic measurement and control, vol.9, pp.44-47, 2001.

[19] J. Yang, J.Y. Yang, H. Ye, "Fisher linear analysis of both theoretical research and its applications", Automation Journal, vol.29, pp.481 -493, 2003. 